\title{
The face inversion effect is not a consequence of aberrant eye movements
}

\author{
Carrick C. Williams \\ Mississippi State University, Mississippi State, Mississippi \\ AND \\ JOHN M. HENDERSON \\ University of Edinburgh, Edinburgh, Scotland
}

\begin{abstract}
The face inversion effect is the finding that inverted faces are more difficult to recognize than other inverted objects. The present study explored the possibility that eye movements have a role in producing the face inversion effect. In Experiment 1, we demonstrated that the faces used here produce a robust face inversion effect when compared with another homogenous set of objects (antique radios). In Experiment 2, participants' eye movements were monitored while they learned a set of faces and during a recognition test. Although we clearly found a face inversion effect, the same features of a face were fixated during the learning and recognition test faces, whether the face was right side up or upside down. Thus, the face inversion effect is not a result of a different pattern of eye movements during the viewing of the face.
\end{abstract}

Faces are among the most important visual objects a person encounters, providing critical information about the age, gender, emotional state, intention, and identity of others. Evidence from a variety of methodologies suggests that face perception is special, supported by dedicated computational and neural processing systems (e.g., Farah, Wilson, Drain, \& Tanaka, 1995; Goren, Sarty, \& Wu, 1975; Kanwisher, McDermott, \& Chun, 1997; McCarthy, Puce, Gore, \& Allison, 1997; but see Gauthier, Tarr, Anderson, Skudlarski, \& Gore, 1999, for a different interpretation). An important source of behavioral evidence for the special nature of face perception is the face inversion effect: Recognition of most visual objects suffers when those objects are inverted, but the inversion deficit is proportionally larger for faces than for other types of visual stimuli (Yin, 1969).

The question we consider in the present study is whether eye movements play any role in producing the face inversion effect. Eye movements are functional in a wide variety of cognitive and perceptual tasks, including reading, picture perception, language processing, and behavioral interaction with the environment (Buswell, 1935; Cutting, Alliprandini, \& Wang, 2000; Hayhoe, Shrivastava, Mruczek, \& Pelz, 2003; Land \& Furneaux, 1997; Land, Mennie, \& Rusted, 1999; Tanenhaus, Spivey-Knowlton, Eberhard, \& Sedivy, 1995; Tinker, 1946; Yarbus, 1967; see Henderson, 2003, 2006; Henderson \& Ferreira, 2004; and Rayner, 1998, for recent reviews). Most research on eye movements during face perception has focused on face recognition (e.g., Althoff \& Cohen, 1999; Luria \& Strauss, 1978; Walker-Smith, Gale,
\& Findlay, 1977). There is considerable evidence that fixations during recognition are predominantly directed to internal facial features, particularly the eyes, nose, and mouth (Althoff \& Cohen, 1999; Luria \& Strauss, 1978; Mertens, Siegmund, \& Grüsser, 1993; Stacey, Walker, \& Underwood, 2005). For example, Walker-Smith et al., (1977) found that fixations tended to be primarily directed to the eyes, nose, and mouth of test faces during both delayed recognition and simultaneous comparison tasks. Groner, Walder, and Groner (1984) reported that the largest proportion of transitions between three facial regions (e.g., fixating the left eye, the right eye, and then the nose) during face perception involved scanning between the two eyes. Henderson, Falk, Minut, Dyer, and Mahadevan (2001) observed that about $60 \%$ of fixation time was spent on the eyes in a face recognition task and that about $90 \%$ of fixation time was spent on the eyes, nose, and mouth.

In a recent study, Henderson, Williams, and Falk (2005) demonstrated that the relationship between eye movements and face perception is functional. In that study, participants were asked to learn unfamiliar faces in preparation for a later memory test. The faces were presented in two blocked conditions. In a free viewing condition, participants were allowed to move their eyes freely as they memorized the faces. In a restricted viewing condition, participants were required to keep their eyes fixated in one central position during learning. Eye movements were monitored in both conditions to ensure that participants followed instructions, and an eye-contingent display change procedure was used to blank the screen if

C.C.Williams, cwilliams@psychology.msstate.edu 
participants strayed from the central position in the restricted viewing condition. The faces were visible for the same total amount of viewing time in the two conditions. Following the learning session, a recognition session was given in which the previously learned faces were mixed with similar new faces, and participants were asked to distinguish old from new. The results showed a clear deficit for the restricted viewing condition. Accuracy rates in the free movement condition were $81 \%$, but were only $52 \%$ when eye movements were prevented during learning. Henderson et al. (2005) also reported that the majority of fixation time was directed to the eyes, nose, and mouth during both face learning and recognition.

The present study was designed to investigate whether a similar functional relationship might exist between fixation patterns and the face inversion effect. Although previous research has demonstrated that face inversion can yield different event-related potentials prior to the movement of the eyes (e.g., Rossion et al., 1999), the possibility exists that later attention to the featural elements of the face - and, consequently, eye movements toward these features - is important to produce a face inversion effect. We reasoned that one consequence of inverting a face might be to make it more difficult to find and orient attention to the critical facial features for recognition: the eyes, nose, and mouth. If this hypothesis is correct, we should find relatively less fixation time on these features in the inverted condition than in the upright condition. Alternatively, the inversion effect might be unrelated to the specific features selected for fixation. This hypothesis is consistent with the idea that the inversion effect is due to disruption of holistic face processing rather than to feature processing. If the latter hypothesis is correct, the same features might be fixated, whether a face is upright or inverted. To test these hypotheses, we had participants learn a set of faces during a learning session, and then tested face recognition for upright and inverted new and old faces. In Experiment 1, we compared recognition of our set of faces against a set of nonface objects (antique radios) to ensure that we could observe the standard face inversion effect in our paradigm. In Experiment 2, we examined eye movements during recognition for upright and inverted faces. To foreshadow the results, although a clear face inversion effect was observed, fixation patterns were very similar for the upright and inverted faces, suggesting that the inversion effect is not a consequence of the inability to direct fixation to relevant face regions.

\section{EXPERIMENT 1}

The purpose of Experiment 1 was to ensure that we could observe the face inversion effect with the specific set of faces, the test conditions, and the display environment to be used in the eyetracking experiment. We compared the inversion effect for our set of faces with a set of antique radios used as control objects. In the learning session, participants viewed 20 faces and 20 antique radios for $10 \mathrm{sec}$ each. During the recognition session, participants viewed the learned faces and radios and 20 new faces and radios. Two recognition conditions were compared: In the upright recognition condition, the faces and radios were displayed in the same upright orientation as in the learning session. In the inverted recognition condition, the faces and radios were displayed upside down. In both cases, participants were required to decide whether the stimulus was new or old, ignoring orientation. Sensitivity measures were compared as a function of orientation.

\section{Method}

Participants. Thirty-two members of the Michigan State University undergraduate participant pool participated in this experiment. All had normal or corrected-to-normal vision and received course credit for participation.

Stimuli. Digitized images of the faces of 40 Michigan State University undergraduate women, all of approximately the same age and of the same ethnicity, served as stimuli (see Henderson et al., 2005). The images, with similar neutral expressions, were obtained with a digital camera under similar lighting conditions. They were imported into Adobe Photoshop, differences in luminance and contrast were equalized, and hair was edited to a uniform style. None of the faces had any overly distinguishing characteristics such as jewelry or idiosyncratic makeup. The resulting images were $400 \times 550$ pixels in size and subtended $7.84^{\circ}$ of visual angle horizontally and $10.78^{\circ}$ vertically at the viewing distance of $1 \mathrm{~m}$, approximately equivalent to the size of a real face (approximately $14 \mathrm{~cm}$ wide) viewed from a distance of $1 \mathrm{~m}$. The faces were embedded in an $800 \times 600$ pixel gray background for presentation. An example face can be found in Henderson et al. (2005) and in Figure 1. The stimuli were presented without the region boundaries and in full color.

Apparatus. The stimuli were displayed at a resolution of $800 \times$ 600 pixels $\times 32,768$ colors on an NEC Multisync XE 15 -in. monitor driven by a Hercules Dynamite Pro super video graphics adapter card.

Procedure. Participants first read a description of the experiment, along with a set of instructions. They were informed that there were two parts to the experiment: a learning session and a recognition session. In the learning session, participants were instructed to study the faces and radios as carefully as possible, to prepare for the recognition test. The nature and difficulty of the test were explained. Following presentation of the 20 faces and 20 radios that were studied in learning, five full-color photographs depicting natural scenes were presented for study. Participants were told to memorize the scenes to prepare for a test that would be given at the end of the experiment. The scenes were included as interpolated filler material to provide a delay between the study and the recognition sessions for the faces and radios. The memory test for the scenes was not given.

In the learning session, the experimenter initiated a trial once the participant had fixated a marker centered on the display. A trial consisted of the presentation of a face or radio in the center of the monitor for $10 \mathrm{sec}$. Presentation of the radios and that of the faces were blocked and were counterbalanced, with half of the participants studying the radios first and the other half studying the faces first.

In the recognition session, the experimenter initiated a trial once the participant had fixated a marker centered on the display, and a face was presented in the center of the monitor until response. There were 40 face recognition trials, 10 (items) $\times 2$ (upright/inverted) $\times$ 2 (old/new), and 40 radio recognition trials, 10 (items) $\times 2$ (upright/ inverted) $\times 2$ (old $/$ new). Items and conditions were presented in a pseudorandom order that was blocked in the same order as the study session. During recognition, participants were told to decide as quickly and as accurately as possible whether or not the displayed item (face or radio) had been presented in the learning session. Participants responded by using two buttons (old or new) on a button box. The entire experiment lasted approximately $45 \mathrm{~min}$. The faces and radios were counterbalanced across conditions via a Latinsquare design, so that all faces and radios appeared in each condition an equal number of times across participants. 


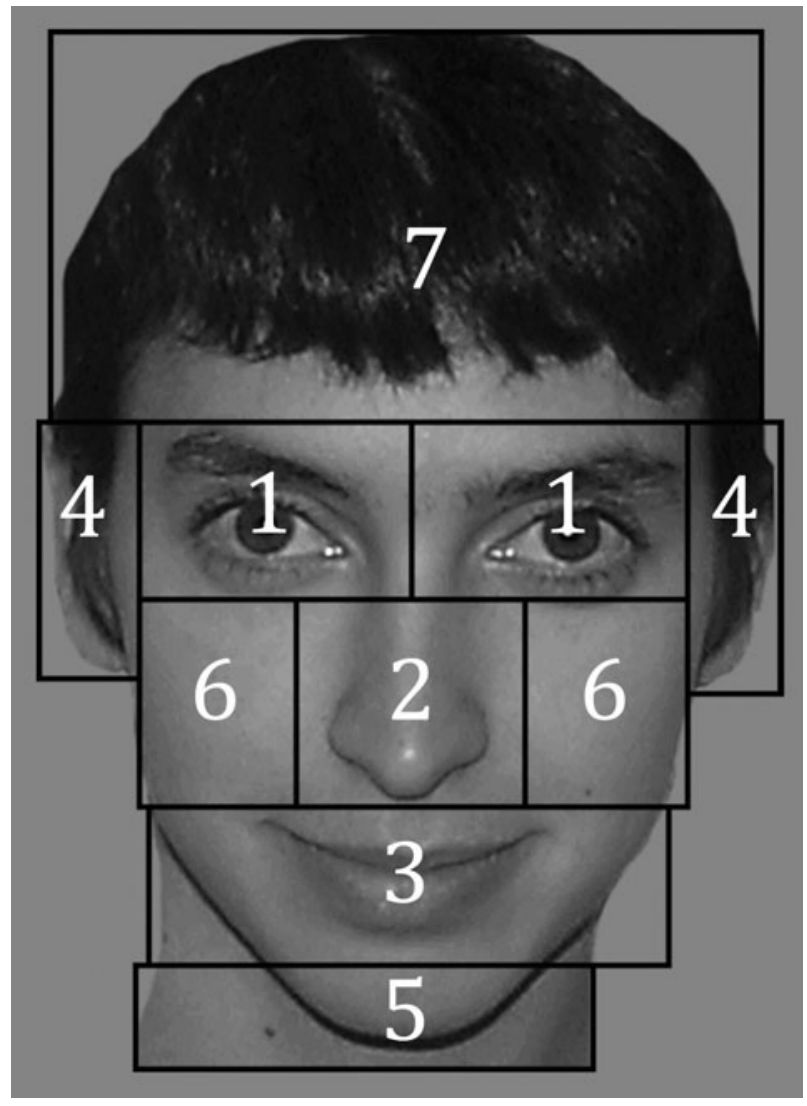

Figure 1. An example of the facial regions that were used in the eyetracking analyses. For the analyses discussed here, we created eye, ear, and cheek regions by collapsing across right and left regions of each feature. The faces were presented in full color and the boundaries were not present in the display.

\section{Results and Discussion}

The main purpose of Experiment 1 was to ensure that a typical face inversion effect can be produced with our stimuli and under our specific viewing conditions. Hit rates, false alarm rates, $A^{\prime}$ (a nonparametric signal detection measure of sensitivity), and response times (RTs) in the recognition session are presented in Table 1 as a function of stimulus type (face or radio) and test orientation (upright or inverted). We focus on $A^{\prime}$ and RT as the most direct and easily interpreted measures of the inversion effect. For $A^{\prime}$, which can be interpreted as equivalent to percent correct in a forced-choice procedure, there was a reliable interaction of stimulus type and orientation $\left[F(1,31)=7.85, p=.009, M S_{\mathrm{e}}=0.018\right]$. As can be seen in Table 1, this interaction reflected the larger inversion effect for faces (.20 difference for upright vs. inverted) than for radios (.06 difference for upright vs. inverted). This threefold increase in the inversion effect for faces over radios represents the paradigmatic face inversion effect. For RT, a similar reliable interaction of stimulus type and orientation was observed $[F(1,31)=10.98, p=$ $\left..002, M S_{\mathrm{e}}=13,815\right]$. As in the $A^{\prime}$ measure, the interaction reflected a much larger inversion effect for faces (337msec difference for upright vs. inverted) than for radios (32-msec difference for upright vs. inverted). In summary, we observed a strong face inversion effect for our stimuli and viewing conditions. We were in a position, therefore, to examine in Experiment 2 the relationship between eye movements and the face inversion effect.

\section{EXPERIMENT 2}

Experiment 2 was designed to determine whether eye movements differ during recognition of upright and inverted faces. Because of the difficulty of keeping participants on the eyetracker for an extended period of time, we excluded the radio condition. Following face learning, face orientation during recognition was manipulated and participants' distributions of fixations were measured. A change in fixation placement as a consequence of face orientation would suggest a functional relationship between eye movements and the face inversion effect. On the other hand, similar fixation patterns for upright and inverted faces would suggest that eye movement patterns are not causally related to the inversion effect.

Participants viewed 20 faces for $10 \mathrm{sec}$ each during a learning session, and were then tested on those faces and 20 new faces in a recognition session. Eye movements were recorded during both learning and recognition. Two recognition conditions were compared. In the upright recognition condition, the faces were displayed in the same orientation as in the learning session. In the inverted recognition condition, the faces were displayed upside down. In both cases, participants were required to decide whether each face was new or old, ignoring orientation. Recognition scores and fixation placement in the recognition session were compared as functions of face orientation.

\section{Method}

Participants. Sixteen members of the Michigan State University undergraduate participant pool who did not participate in Experiment 1 were recruited for this experiment. All had normal, uncorrected vision, and received course credit for participation.

Stimuli. The stimuli were the same 40 digitized images of faces used in Experiment 1.

Apparatus. The stimuli were displayed in the same way as in Experiment 1. Eye movements were monitored using a Generation 5.5 Stanford Research Institute Dual Purkinje Image Eye Tracker (Crane, 1994), which has a resolution of $1 \mathrm{~min}$ of arc and a linear output over the range of the visual display used. A bite bar and forehead rest maintained the participant's viewing position and distance. The right eye was tracked, though viewing was binocular. Signals were sampled from the eyetracker using the polling mode of the

Table 1

Results From Experiment 1

\begin{tabular}{lrrrrrrr}
\hline & \multicolumn{3}{c}{ Accuracy } & & \multicolumn{3}{c}{ Reaction Time* $^{*}$} \\
\cline { 2 - 4 } & HR & FAR & $A^{\prime}$ & & Studied & New & Mean \\
\hline Upright & .775 & .097 & .900 & & 1,297 & 1,366 & 1,331 \\
Inverted & .722 & .425 & .705 & & 1,488 & 1,848 & 1,668 \\
& & & Radios & & & \\
Upright & .819 & .278 & .835 & & 1,449 & 1,681 & 1,562 \\
Inverted & .712 & .306 & .772 & & 1,472 & 1,715 & 1,594 \\
\hline
\end{tabular}

Note-HR, hit rate; FAR, false alarm rate. ${ }^{*}$ Correct responses in milliseconds. 
Table 2

Response Measures for Experiment 2

\begin{tabular}{lccccccc}
\hline & \multicolumn{3}{c}{ Accuracy } & & \multicolumn{3}{c}{ Reaction Time* } \\
\cline { 2 - 4 } \cline { 7 - 9 } & HR & FAR & $A^{\prime}$ & & Studied & New & Mean \\
\hline Upright & .713 & .119 & .875 & & 2,010 & 2,020 & 2,015 \\
Inverted & .724 & .391 & .742 & & 2,435 & 2,714 & 2,574 \\
\hline
\end{tabular}

Note-HR, hit rate; FAR, false alarm rate. ${ }^{*}$ Correct responses in milliseconds.

Data Translations DT2803 analog-to-digital converter, producing a sampling rate slightly faster than $1000 \mathrm{~Hz}$.

Procedure. Participants first read a description of the experiment along with a set of instructions. A bite bar was prepared, the experimental task was explained, and questions were answered. Participants were informed that there were two parts to the experiment: a learning session and a recognition session. In the learning session, participants were instructed to study the faces as carefully as possible to prepare for the recognition test. The nature and difficulty of the test was explained. Following presentation of the 20 faces that were studied, five full-color photographs depicting natural scenes were presented for study. Participants were told to memorize the scenes to prepare for a test that would be given at the end of the experiment. The scenes were again included as interpolated filler material to provide a delay between the study and the recognition sessions for the faces. As in Experiment 1, the memory test for the scenes was not given.

To calibrate the eyetracker, participants fixated a series of markers presented on the display monitor. Calibration was checked by displaying a fixation marker indicating the computer's estimate of the current fixation position. Calibration was considered accurate when the estimated fixation position was within \pm 5 min of arc of each of five displayed test locations, and was checked periodically throughout the experiment. The eyetracker was recalibrated whenever this criterion was not met.

Rather than starting the learning session trials with the participant fixated in the center of the face, as was done in Experiment 1, the learning trials in Experiment 2 were initiated when the participant was fixated on a marker on the left side of the computer screen not overlapping any portion of the presented face. The participant was, therefore, required to execute a rightward eye movement to fixate the features of the face. A trial consisted of the presentation of a face in the center of the monitor for $10 \mathrm{sec}$. Similarly, in the recognition session, the experimenter initiated a trial once the participant had fixated a marker on the left side of the screen, and a face was presented in the center of the monitor until response, or for $10 \mathrm{sec}$ maximum. In the recognition session, there were 40 recognition trials, 10 (faces) $\times 2$ (upright/inverted) $\times 2$ (old $/$ new $)$. Conditions were presented in a pseudorandom order. During recognition, participants were told to decide as quickly and as accurately as possible whether or not the face had been presented in the learning session. Participants responded by pressing two buttons (old or new) on a button box. The entire experiment lasted approximately $35 \mathrm{~min}$. The faces were counterbalanced across conditions via a Latin-square design, so that all faces appeared in each condition an equal number of times across participants.

Table 3

Global Measures of the Eye Movements Performed During Experiment 2

\begin{tabular}{lccc}
\hline & \multicolumn{3}{c}{ Performed During Experiment 2 } \\
\hline & & $\begin{array}{c}\text { Avg. Fixation } \\
\text { Duration }\end{array}$ & $\begin{array}{c}\text { Avg. Saccade } \\
\text { Amplitude }\end{array}$ \\
\hline \multirow{2}{*}{ Old } & Upright & 260 & 2.08 \\
& Inverted & 260 & 2.07 \\
& Upright & 260 & 2.16 \\
& Inverted & 264 & 2.09 \\
\hline
\end{tabular}

\section{Results and Discussion}

To establish that the inverted faces were more difficult to identify than the upright faces in the present experiment, we computed hit rates, false alarm rates, $A^{\prime}$, and RTs in the recognition session (see Table 2). As in Experiment 1 , statistical analyses focused on $A^{\prime}$ and RT. For $A^{\prime}$, there was a reliable .133 effect of orientation $[F(1,15)=$ $15.69, p=.001, M S_{\mathrm{e}}=0.009$ ], with inverted faces being more difficult to recognize than upright faces. Mean RTs were consistent with these results, with reliably slower responses, by $559 \mathrm{msec}$, for the inverted faces than for the upright faces $\left[F(1,15)=20.31, p<.001, M S_{\mathrm{e}}=0.246\right]$.

The central question in the present study was whether the face inversion effect is related to changes in fixation placement; if so, fixation patterns should differ in the upright and inverted recognition conditions. We began by looking at mean fixation duration and mean saccade amplitude to determine whether there were overall differences in the ways in which the upright and inverted faces were viewed. The values of these measures for the old and new upright and inverted faces are shown in Table 3. As can be seen, the values for these measures were very similar across conditions. The average fixation duration was $261 \mathrm{msec}$ and did not differ by condition $(F<1)$. Average saccade amplitude was $2.1^{\circ}$ and did not differ by condition either $\left[F(1,15)=1.58, p>.20, M S_{\mathrm{e}}=0.028\right]$.

To quantify the dispersion of eye fixations over the features of the faces, each face was divided into seven nonoverlapping rectangular regions (see Henderson et al., 2001; Henderson et al., 2005; Minut, Mahadevan, Henderson, \& Dyer, 2000). These features corresponded to the following principal facial features: (1) eyes, (2) nose, (3) mouth, (4) ears, (5) chin and neck, (6) cheeks, and (7) forehead and top of head. The regions were uniquely defined for each face used in the experiment (i.e., each of the 40 faces used had its own facial region map; see Figure 1 for an example) and were the smallest rectangle that could completely enclose the feature. To determine whether the features selected for fixation differed for upright and inverted faces, we compared the amount of time spent on the seven defined facial regions in the two orientation conditions. A direct comparison of fixation time on specific regions in the upright and inverted recognition sessions is problematic, because the faces were viewed for different amounts of time in the two conditions (see Table 2). To control for this difference in viewing time, but to still allow for comparison across conditions, fixation time in each region was calculated as a proportion of total fixation time for that trial (see Henderson et al., 2005). Figure 2 shows proportion of viewing time as a function of stimulus orientation and face region for the recognition session. The patterns of results were similar for the studied versus the new faces, with only one significant main effect of previously viewing the face [the mouth region, with a greater proportion of time being devoted to the mouth for new faces $\left[F(1,15)=6.47, p=.022, M S_{\mathrm{e}}=0.001\right]$ and only one interaction between orientation and previously viewing the face [the cheek region, $F(1,15)=6.53, p=$ $\left..022, M S_{\mathrm{e}}=0.001\right]$. No other effects for the seven face 


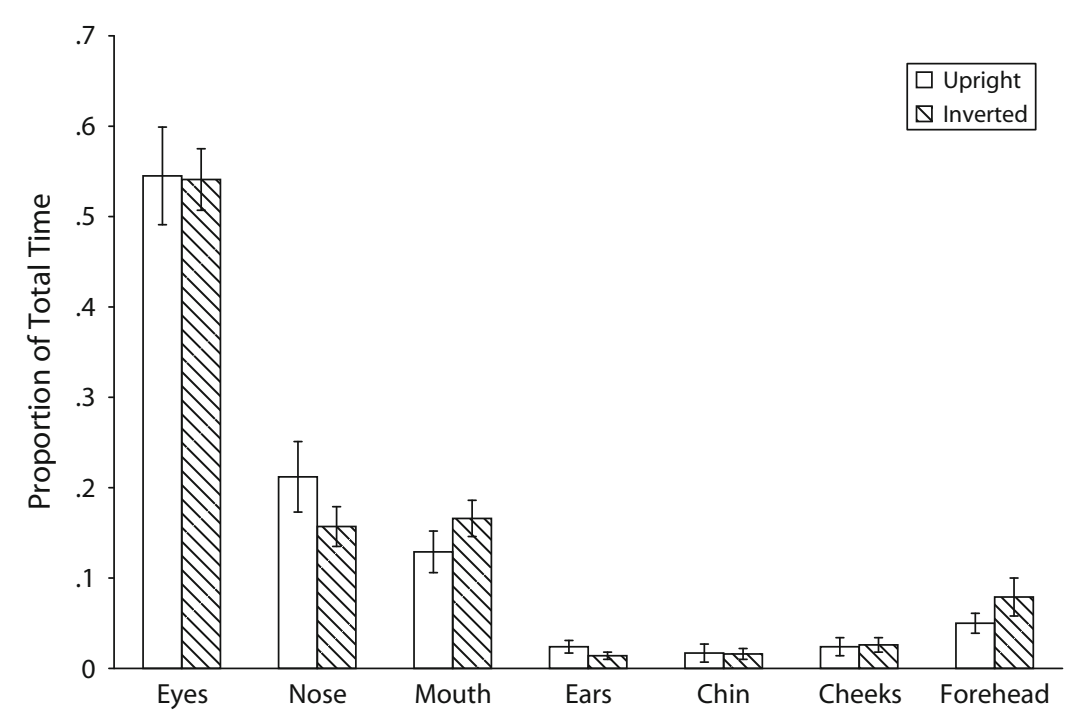

Figure 2. Proportion of total time spent on each facial feature for each orientation during the recognition test of Experiment 2. Error bars represent the standard errors of the means.

regions were reliable $\left(F_{\mathrm{S}}<1.1\right)$. Given the limited impact of previously viewing the face on the proportion of time spent in the different facial regions, we will not discuss this factor further here.

As can be seen in Figure 2, the proportion of fixation time spent on each region was remarkably similar in the upright and inverted conditions. Overall, the rank ordering of the proportion of fixation time spent over the features of the faces was similar across conditions, with the majority (over 50\%) of time spent on the eyes. The nose and mouth received a large proportion of the remainder of the fixation time. This focus on the eyes, nose, and mouth replicates the results of Henderson et al. (2005) with this set of faces, as well as earlier studies cited in the introduction. This analysis once again shows the dominance of the eyes as an important feature for both face learning and recognition.

There was a hint in the data that proportionally less fixation time was spent on the nose and more on the mouth in the inverted than in the upright recognition conditions, but these effects were small, not statistically reliable, and within the amount of variation typically observed with these stimuli (Henderson et al., 2005). As a liberal test of these data, we compared the mean proportion of total time in each region as a function of inversion in the recognition session uncorrected for the dependencies in the data and without correction for the inflated Type I error rate. None of these analyses showed significant differences: for the eye, chin, and cheek regions $(F \mathrm{~s}<1)$; for the nose region $\left[F(1,15)=2.13, p>.15, M S_{\mathrm{e}}=0.022\right]$; for the mouth region $\left[F(1,15)=2.72, p>.10, M S_{\mathrm{e}}=0.008\right]$; for the ear region $\left[F(1,15)=1.87, p>.15, M S_{\mathrm{e}}=0.001\right]$; and for the forehead region $\left[F(1,15)=1.68, p>.20, M S_{\mathrm{e}}=0.001\right]$.

We also examined several other measures of the distribution of fixations over faces, including the proportion of the number of fixations in each region and the probability of entering each region. Both of these analyses mirrored those from the fixation time analysis. As can be seen in
Figure 3, the proportions of fixations during the recognition test for the upright and inverted conditions were extremely similar, and there were no main effects of orientation for any of the facial regions [nose region, $F(1,15)=$ $2.14, p>.15, M S_{\mathrm{e}}=0.021$; mouth region, $F(1,15)=$ $1.32, p>.20, M S_{\mathrm{e}}=0.008$; forehead region, $F(1,15)=$ $1.89, p>.15, M S_{\mathrm{e}}=0.009$; all other regions, $\left.F \mathrm{~s}<1\right]$.

A similar pattern was found for the mean proportion of trials, in which each feature region was fixated at least once per face. As can be seen in Figure 4, on almost every trial participants fixated the eyes and most participants fixated the nose and mouth. The forehead also received a relatively high proportion of fixations. The heavy emphasis on these features is similar to the data observed in our earlier learning study (Henderson et al., 2005), as well as in studies of eye movements during recognition (Groner et al., 1984; Henderson et al., 2001; Henderson et al., 2005; WalkerSmith et al., 1977). The only significant effect of face orientation on the proportion of regions viewed was on the mouth region $\left[F(1,15)=4.78, p=.045, M S_{\mathrm{e}}=0.095\right]$, with that region being viewed on a greater proportion of trials when the face was inverted. For all other regions, the orientation of the face had no effect on the likelihood of viewing the feature [eye region, $F(1,15)=2.26, p>.15$, $M S_{\mathrm{e}}=0.037$; ear region, $F(1,15)=1.12, p>.20, M S_{\mathrm{e}}=$ 0.011 ; forehead region, $F(1,15)=1.33, p>.20, M S_{\mathrm{e}}=$ 0.062 ; all other regions, $\left.F_{\mathrm{S}}<1\right] .^{1}$

The previous analyses indicate that the viewing patterns of the facial regions across an entire trial were similar for the upright and inverted faces. We also examined whether orientation affected the scan pattern within a trial. In order to accomplish this analysis, we analyzed by ordinal fixation the proportion of fixations targeted to a facial region for both upright and inverted faces during the recognition test. Because the test stimuli were terminated by the participant when he or she made a decision, we could only analyze fixations $2-7$ (starting with the second fixation, 


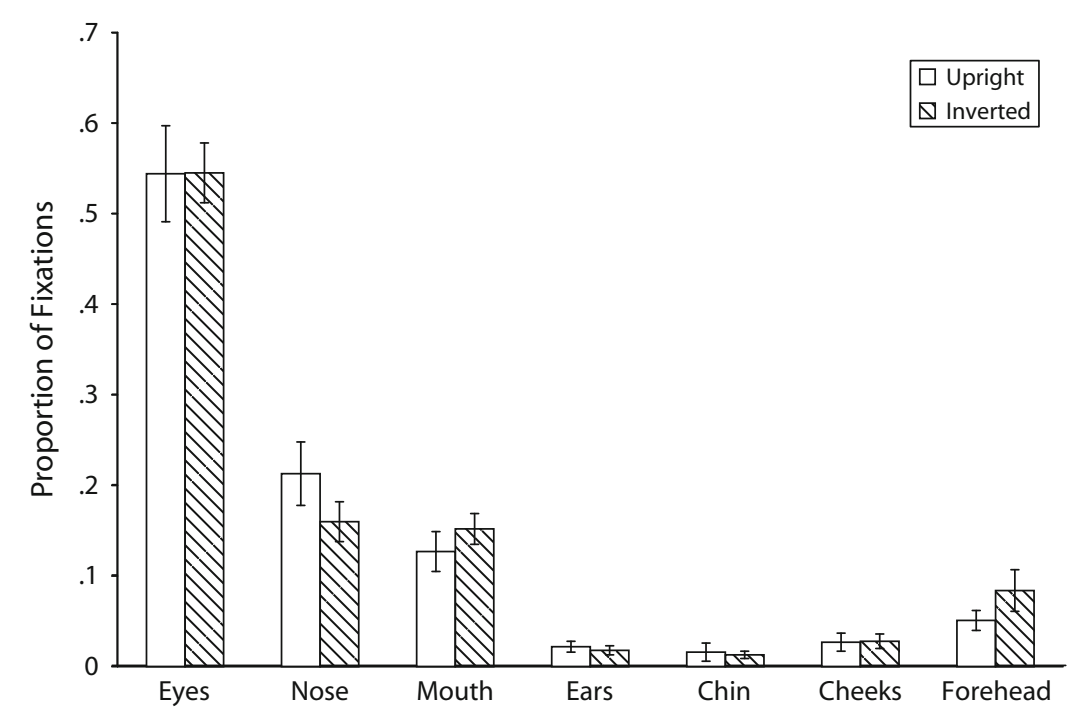

Figure 3. Proportion of fixations on each facial feature for each orientation during the recognition test of Experiment 2. Error bars represent the standard errors of the means.

since the first fixation was to the left of the face), creating a 2 (orientation) $\times 7$ (facial region) $\times 6$ (ordinal fixation) interaction analysis. Additionally, in one condition, one participant did not make six fixations on the face, and thus was removed from this analysis. Critically, we found no interaction of orientation in the likelihood of fixating facial regions across the ordinal fixations $(F<1)$, indicating that across the trial the upright and inverted faces are viewed similarly (see Table 4). Overall, these analyses indicate that eye movements differ little when upright and inverted faces are recognized.

Finally, we examined the mean proportion of the total amount of time each feature was fixated in the learning phase and in the recognition session for the four types of stimuli (upright and inverted new and old faces). In our prior study, we found very similar eye movements during learning and recognition. Mean fixation duration was $321 \mathrm{msec}$, and mean saccade length was $1.89^{\circ}$ during learning. These values are similar to those of $318 \mathrm{msec}$ and $2.09^{\circ}$ reported by Henderson et al. (2005) for learning these same faces. As shown in Figure 5, participants devoted the majority (over $50 \%$ ) of fixation time to the eyes. Figure 6 shows a typical scan pattern of fixations and saccades made on a single face by a single participant during the learning session. It is also clear from the pattern in Figure 5 that the distribution of fixation time

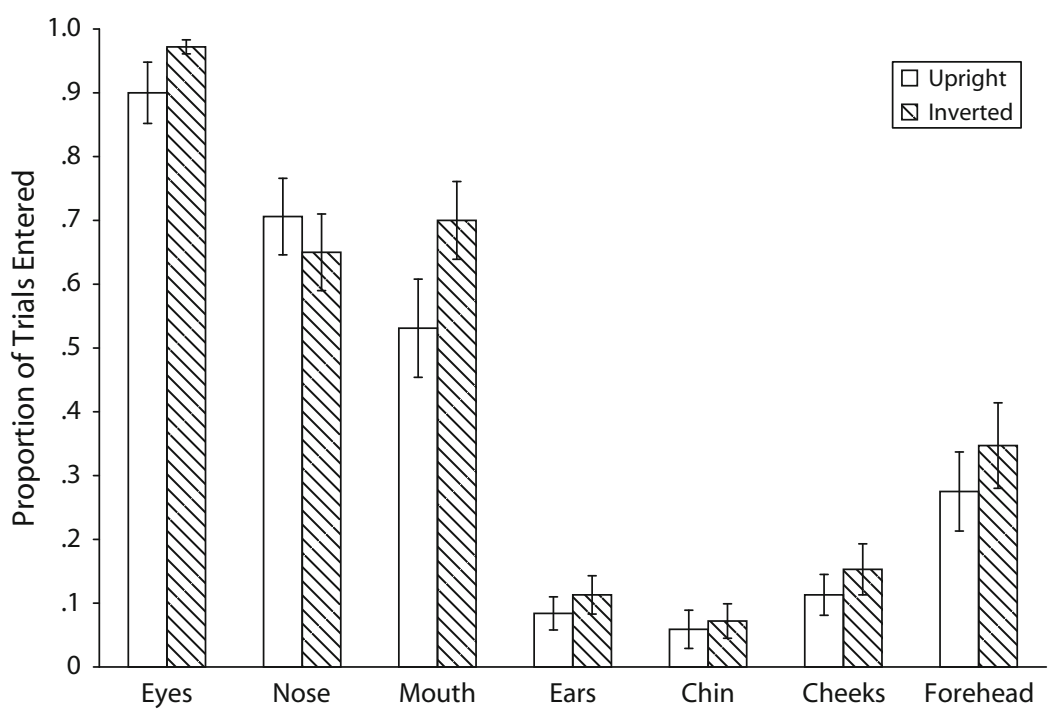

Figure 4. Proportion of trials on which each facial feature was viewed for each orientation during the recognition test of Experiment 2. Error bars represent the standard errors of the means. 
Table 4

Scan Pattern Data

\begin{tabular}{|c|c|c|c|c|c|c|c|c|c|c|c|c|}
\hline & \multicolumn{12}{|c|}{ Ordinal Fixation } \\
\hline & \multicolumn{2}{|c|}{2} & \multicolumn{2}{|c|}{3} & \multicolumn{2}{|c|}{4} & \multicolumn{2}{|c|}{5} & \multicolumn{2}{|c|}{6} & \multicolumn{2}{|c|}{7} \\
\hline & $M$ & $S E$ & $M$ & $S E$ & $M$ & $S E$ & $M$ & $S E$ & $M$ & $S E$ & $M$ & $S E$ \\
\hline \multicolumn{13}{|c|}{ Upright Faces } \\
\hline Eyes & .62 & .10 & .60 & .10 & .42 & .07 & .46 & .06 & .52 & .07 & .53 & .06 \\
\hline Nose & .23 & .06 & .25 & .08 & .28 & .07 & .21 & .06 & .18 & .04 & .18 & .04 \\
\hline Mouth & .08 & .04 & .10 & .04 & .22 & .06 & .15 & .03 & .15 & .03 & .08 & .03 \\
\hline Ears & .00 & .00 & .00 & .00 & .01 & .01 & .03 & .02 & .01 & .00 & .03 & .02 \\
\hline Chin & .01 & .01 & .03 & .02 & .02 & .01 & .01 & .00 & .04 & .03 & .04 & .03 \\
\hline Cheek & .05 & .03 & .01 & .01 & .01 & .01 & .03 & .02 & .02 & .01 & .00 & .00 \\
\hline Forehead & .00 & .00 & .00 & .00 & .03 & .01 & .08 & .02 & .08 & .04 & .10 & .02 \\
\hline \multicolumn{13}{|c|}{ Inverted Faces } \\
\hline Eyes & .69 & .07 & .69 & .07 & .50 & .06 & .47 & .04 & .53 & .04 & .45 & .06 \\
\hline Nose & .24 & .07 & .14 & .04 & .18 & .04 & .15 & .03 & .12 & .04 & .13 & .04 \\
\hline Mouth & .02 & .01 & .10 & .04 & .20 & .04 & .21 & .04 & .13 & .03 & .19 & .03 \\
\hline Ears & .00 & .00 & .00 & .00 & .00 & .00 & .00 & .00 & .01 & .01 & .02 & .02 \\
\hline Chin & .00 & .00 & .00 & .00 & .01 & .01 & .01 & .01 & .02 & .01 & .03 & .01 \\
\hline Cheek & .02 & .02 & .03 & .02 & .02 & .01 & .02 & .01 & .04 & .02 & .02 & .01 \\
\hline Forehead & .01 & .01 & .05 & .04 & .08 & .03 & .11 & .03 & .14 & .04 & .15 & .04 \\
\hline
\end{tabular}

Note-The data in the table are the proportions of fixations, with standard errors, devoted to the individual features for upright and inverted faces during the recognition test. Fixations start with the second fixation on the face, because the first fixation was to the left of the face.

across the facial features was very similar in learning and recognition, regardless of orientation; that is, the distribution of fixation time did not become more restricted from learning to recognition as has been reported previously [interaction of facial region and learning and recognition condition, $\left[F(24,360)=1.14, p>.20, M S_{\mathrm{e}}=0.005\right]$.

\section{GENERAL DISCUSSION}

The present study was designed to determine whether a functional relationship exists between eye movements during face recognition and the face inversion effect. Participants first learned a set of faces, and then were asked to recognize those faces in either an upright or an inverted orientation. In Experiment 1, a clear face inversion effect was found for learned faces versus another learned set of relatively homogeneous objects. In Experiment 2, eye movements were recorded during both learning and recognition of the faces. Participants focused most of their fixations on the main features of the faces - eyes, nose, and mouth - during both learning and recognition. Furthermore, though inverted faces were again more difficult to recognize than were upright faces, fixation time was distributed over the same set of features during learning and recognition and during recognition of upright and inverted faces. This represents the first direct evidence that patterns of eye fixations are unrelated to the face inversion effect.

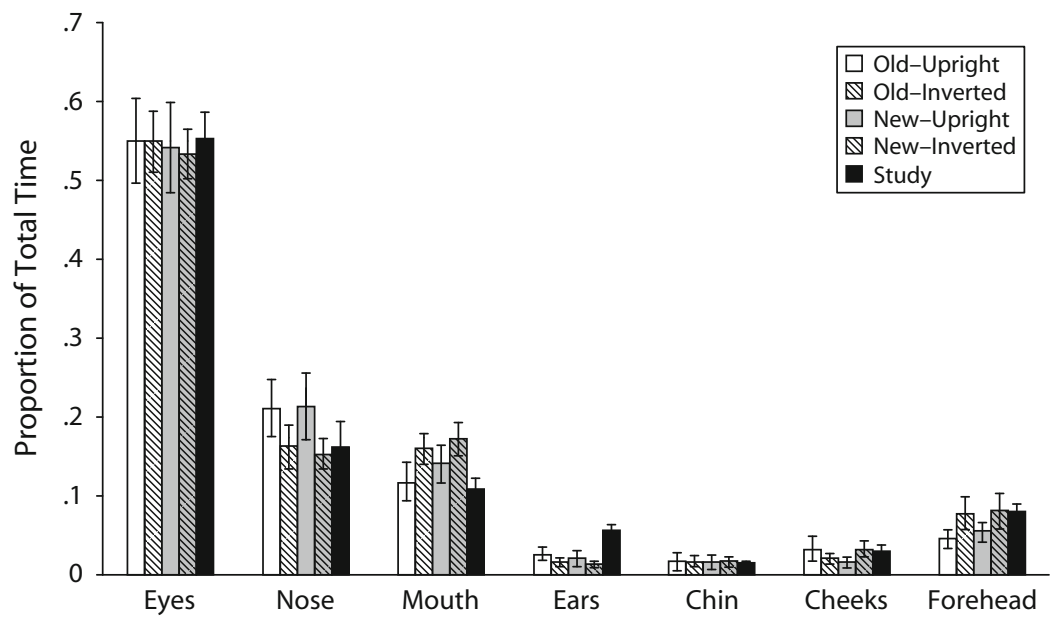

Figure 5. Proportion of total time spent on each facial feature for the learning session and each condition during the recognition test of Experiment 2. Error bars represent the standard errors of the means. 


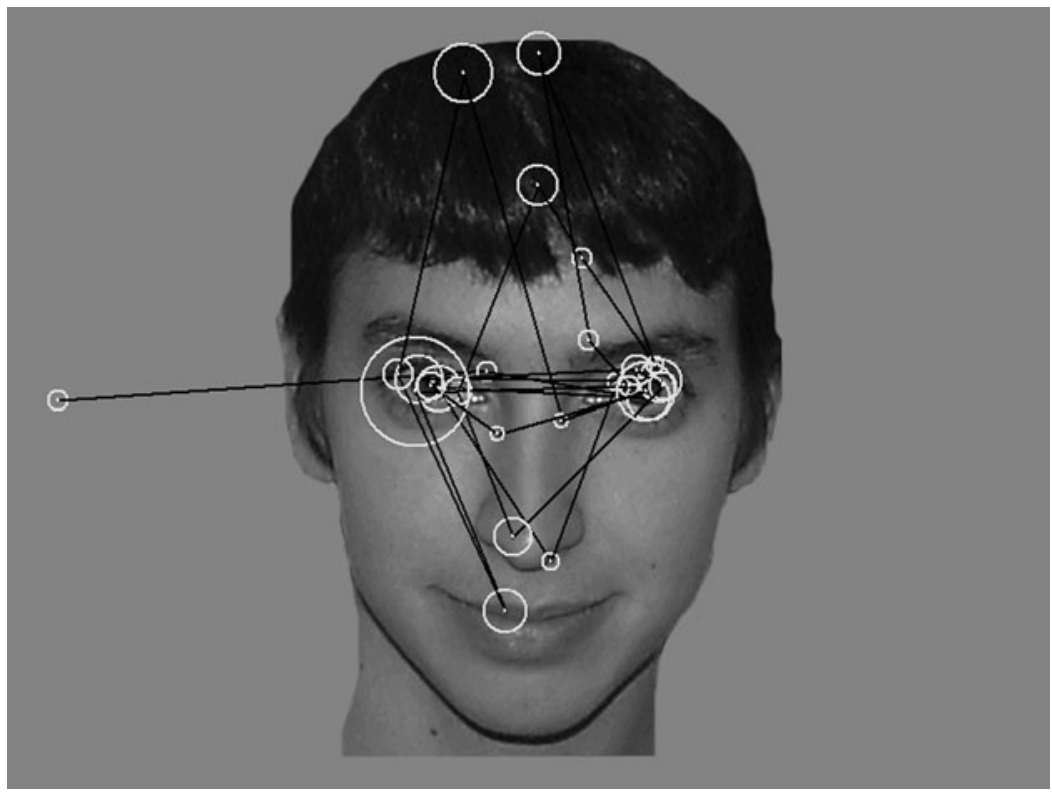

Figure 6. An example scan pattern for one participant on one face during the learning session. The circles represent the location of the fixations and the diameter of the circle is proportional to the fixation duration for that fixation.

If faces are processed holistically, what role might eye movements play in the learning process? One possibility is that initial encoding of relations among features benefits from foveal analysis. For example, determining the distance between the eyes in a face, or the relationship between the distance between the eyes in a particular face and the average distance between eyes in faces in general (i.e., second-order relations), may benefit from fixation on, or near, those features. A second possibility is that the eye movements themselves are functional in determining relations among features. For example, the length of a saccade between two eyes provides direct information about the distance between those eyes. A third possibility is that encoding of specific feature detail during learning is important for achieving a high level of recognition performance for faces that are not highly overlearned.

Alternatively, Sekuler, Gaspar, Gold, and Bennett (2004) have claimed that processing differences for inverted and upright faces relate to the efficiency of the extraction of information rather than to a qualitative difference in the processing strategy. Our results indicating that there are no differences in the eye movement patterns do not provide evidence for a difference in the efficiency of extraction of information for upright and inverted faces. However, Sekuler et al. did find that the same areas of the face were used in their face discrimination task for both upright and inverted faces. Similarly, we found that the same parts of the face were viewed regardless of the face's orientation. Additional studies will be required to tease these possibilities apart. In any case, it is clear that the information used to encode faces, whether it is holistic or not, is not simply derived from low-spatial frequency analysis taking place solely outside of foveal vision.
This study also provides additional information concerning two related issues. First, in contrast to Yarbus (1967), but consistent with other more recent eyemovement studies of face processing (e.g., Althoff \& Cohen, 1999; Henderson et al., 2001; Henderson et al., 2005; Walker-Smith et al., 1977), we found that fixation was directed predominantly to internal facial features during recognition. The present study extends these results to both old and new inverted faces during recognition. Second, Althoff and Cohen (1999) reported a greater concentration of feature selection on fewer critical features in face recognition than in face learning. Henderson et al. (2005) found a similar pattern in their study of the functional role of eye movements during learning, with proportionally more concentration of fixation time on the critical features during recognition than during learning. However, contrary to the idea that this restriction in feature selection is due to prior exposure and therefore familiarity with the older faces, Henderson et al. (2005) found a similar concentration of time on the critical features in the recognition session for both the previously learned and the new distractor faces, suggesting that the concentration of feature selection was a task effect rather than a familiarity effect. In the present study, we found no change in the concentration of fixation time on the critical features from learning to recognition for either old or new faces, whether they were upright or inverted. Together, the results suggest that there is no necessary relationship between the degree of feature sampling and face familiarity, contrary to Althoff and Cohen (1999). Although this change in fixation concentration can be found, it is not necessary, and so does not invariably index familiarity during the recognition processes. 


\section{AUTHOR NOTE}

This research was supported by the Army Research Office (W911NF04-1-0078). The opinions expressed in this article are those of the authors and do not necessarily represent the views of the Department of the Army or any other governmental organization. We thank Richard Falk for his contributions to this research. Portions of this research were presented at the 22nd annual meeting of the Cognitive Science Society. Correspondence concerning this article should be addressed to C. C. Williams, Department of Psychology, Mississippi State University, Mississippi State, MS 39762 (e-mail: cwilliams@psychology.msstate.edu) or J. M. Henderson, Psychology, PPLS, University of Edinburgh, Edinburgh, EH8 9JZ, Scotland (e-mail: john.m.henderson@ed.ac.uk).

\section{REFERENCES}

Althoff, R. R., \& Cohen, N. J. (1999). Eye-movement-based memory effect: A reprocessing effect in face perception. Journal of Experimental Psychology: Learning, Memory, \& Cognition, 25, 9971010.

Buswell, G. T. (1935). How people look at pictures. Chicago: University of Chicago Press.

Crane, H. D. (1994). The Purkinje image eyetracker, image stabilization, and related forms of stimulus manipulation. In D. H. Kelly (Ed.), Visual science and engineering: Models and applications (pp. 15-89). New York: Marcel Dekker.

Cutting, J. E., Alliprandini, P. M. Z., \& Wang, R. F. (2000). Seeking one's heading through eye movements. Psychonomic Bulletin \& Review, 7, 490-498.

Farah, M. J., Wilson, K. D., Drain, H. M., \& Tanaka, J. R. (1995). The inverted face inversion effect in prosopagnosia: Evidence for mandatory, face-specific perceptual mechanisms. Vision Research, 35, 2089-2093.

Gauthier, I., Tarr, M. J., Anderson, A. W., Skudlarski, P., \& Gore, J. C. (1999). Activation of the middle fusiform "face area" increases with expertise in recognizing novel objects. Nature Neuroscience, 2, 568-573.

Goren, C. C., Sarty, M., \& Wu, P. Y. K. (1975). Visual following and pattern discrimination of face-like stimuli by newborn infants. Pediatrics, 56, 544-549.

Groner, R., Walder, F., \& Groner, M. (1984). Looking at faces: Local and global aspects of scanpaths. In A. G. Gale \& F. Johnson (Eds.), Theoretical and applied aspects of eye movement research (pp. 523-533). New York: Elsevier.

Hayhoe, M. M., Shrivastava, A., Mruczek, R., \& Pelz, J. B. (2003). Visual memory and motor planning in a natural task. Journal of Vision, 3, 49-63.

Henderson, J. M. (2003). Human gaze control during real-world scene perception. Trends in Cognitive Sciences, 7, 498-504.

Henderson, J. M. (2006). Eye movements. In C. Senior, T. Russell, \& M. Gazzaniga (Eds.), Methods in mind (pp. 171-191). Cambridge, MA: MIT Press.

Henderson, J. M., Falk, R. J., Minut, S., Dyer, F. C., \& MahadeVAN, S. (2001). Gaze control for face learning and recognition by humans and machines. In T. Shipley \& P. Kellman (Eds.), From fragments to objects: Segmentation processes in vision (pp. 463-482). Amsterdam: Elsevier.

Henderson, J. M., \& Ferreira, F. (2004). Scene perception for psycholinguists. In J. M. Henderson \& F. Ferreira (Eds.), The interface of language, vision, and action: Eye movements and the visual world (pp. 1-58). New York: Psychology Press.

Henderson, J. M., Williams, C. C., \& Falk, R. J. (2005). Eye movements are functional during face learning. Memory \& Cognition, 33, 98-106.

Kanwisher, N., McDermott, J., \& Chun, M. M. (1997). The fusiform face area: A module in human extrastriate cortex specialized for face perception. Journal of Neuroscience, 17, 4302-4311.

LAND, M. F., \& Furneaux, S. (1997). The knowledge base of the oculomotor system. Philosophical Transactions of the Royal Society of London, Series B: Biological Sciences, 352, 1231-1239.

Land, M. F., Mennie, N., \& Rusted, J. M. (1999). The roles of vision and eye movements in the control of activities of daily living. Perception, 28, 1311-1328.

LuRIa, S. M., \& STRAuss, M. S. (1978). Comparison of eye movements over faces in photographic positives and negatives. Perception, 7, 349358.

McCarthy, G., Puce, A., Gore, J. C., \& Allison, T. (1997). Facespecific processing in the human fusiform gyrus. Journal of Cognitive Neuroscience, 9, 605-610.

Mertens, I., Siegmund, H., \& Grüsser, O. J. (1993). Gaze motor asymmetries in the perception of faces during a memory task. Neuropsychologia, 31, 989-998.

Minut, S., Mahadevan, S., Henderson, J. M., \& Dyer, F. (2000). Face recognition using foveal vision. In S.-W. Lee, H. H. Bulthoff, \& T. Poggio (Eds.), Biologically motivated computer vision. Berlin: Springer.

RAYNER, K. (1998). Eye movements in reading and information processing: 20 years of research. Psychological Bulletin, 124, 372-422.

Rossion, B., Delvenne, J.-F., Debatisse, D., Goffaux, V., Bruyer, R., Crommelinck, M., \& GuÉrit, J. M. (1999). Spatio-temporal localization of the face inversion effect: An event-related potentials study. Biological Psychology, 50, 173-189.

Sekuler, A. B., Gaspar, C. M., Gold, J. M., \& Bennett, P. J. (2004). Inversion leads to quantitative, not qualitative, changes in face processing. Current Biology, 14, 391-396.

Stacey, P. C., Walker, S., \& Underwood, J. D. M. (2005). Face processing and familiarity: Evidence from eye-movement data. British Journal of Psychology, 96, 407-422.

Tanenhaus, M. K., Spivey-Knowlton, M. J., Eberhard, K. M., \& Sedivy, J. C. (1995). Integration of visual and linguistic information in spoken language comprehension. Science, 268, 1632-1634.

Tinker, M. A. (1946). The study of eye movements in reading. Psychological Bulletin, 43, 93-120.

Walker-Smith, G. J., Gale, A. G., \& Findlay, J. M. (1977). Eye movement strategies involved in face perception. Perception, 6, 313-326.

Yarbus, A. (1967). Eye movements and vision. New York: Plenum.

YIN, R. K. (1969). Looking at upside-down faces. Journal of Experimental Psychology, 81, 141-145.

\section{NOTE}

1. As a further evaluation of the patterns of eye movements, we also examined the interaction of the facial region with the facial orientation for the three measures discussed. The results of these analyses indicate no difference in the patterns of the fixations for the proportion of fixation time $(F<1)$, the proportion of fixations $(F<1)$, and the proportion of facial regions entered $\left[F(6,90)=1.46, p=.20, M S_{\mathrm{e}}=.052\right]$. Although the overall interactions were not significant, limiting the analysis to only the nose and mouth regions, we found interactions of orientation and facial regions for the proportion of fixations time $[F(1,15)=5.70$, $\left.p=.03, M S_{\mathrm{e}}=.012\right]$, proportion of fixations $[F(1,15)=4.85, p=.04$, $\left.M S_{\mathrm{e}}=.010\right]$, and proportion of facial regions entered $[F(1,15)=5.44$, $\left.p=.03, M S_{\mathrm{e}}=.075\right]$. Inverting a face appears to decrease the likelihood of fixating the nose region while simultaneously increasing the likelihood of fixating the mouth region. The finding of increased viewing of the mouth cannot be attributed to the possibility that the mouth region of the inverted faces occupies the location of the eyes on an upright face. In fact, both the nose and mouth regions tend to overlap with the area occupied by the forehead region when the face is upright. Because this comparison was one of 21 possible post hoc pairwise comparisons for the different facial regions, it is important to treat the finding of a difference with caution, and to view any explanation as speculative; however, this pattern could indicate that the relative importance of these regions is changed when the face is inverted. When the faces were inverted, the proportion of total time and proportion of fixations were both equivalent for the nose and the mouth regions. As can be seen in Table 4, the main difference between the upright and the inverted faces is that within the first 7 fixations in the trial, participants look less at the nose region; there is no dramatic increase in viewing the mouth region. Thus, the increase in the viewing of the mouth region must occur when the inverted face is viewed for a relatively longer period of time. Overall, because we found no overall difference in the pattern of viewing upright and inverted faces, it is important to view this particular interaction with caution, but it could indicate that there is a subtle alteration of the viewing pattern when a face is inverted.

(Manuscript received July 25, 2006; revision accepted for publication February 8, 2007.) 